\title{
More success for immunotherapy with nivolumab approval for metastatic RCC
}

$\mathrm{F}$ ollowing its recent success in non-small-cell lung cancer (NSCLC), the immunotherapeutic agent nivolumab received approval from the US Food and Drug Administration last fall for the treatment of advanced clear-cell renal-cell carcinoma (RCC) after prior antiangiogenic therapy. Nivolumab, an immune checkpoint inhibitor, is a fully human immunoglobulin G4 monoclonal antibody that binds to the programmed cell death 1 (PD-1) receptor on the surface of $T$ cells, prevents their inactivation by tumor cells overexpressing PD-1 ligands, and helps to reinstate the anti-tumor immune response.

The approval was based on the results of the openlabel, randomized, phase 3 CheckMate-025 trial, in which nivolumab became the first drug to demonstrate an overall survival (OS) benefit in patients with advanced RCC in the second-line setting, and marks the third tumor type for which nivolumab is now indicated, the others being NSCLC and melanoma.

The trial was carried out during October 2012-March 2014 at 146 sites in 24 countries. A total of 821 patients aged 18 years or older, with histologically confirmed advanced, clear-cell RCC, measurable disease per Response Evaluation Criteria in Solid Tumors (version 1.1), a Karnofsky Performance Status of at least 70 (cares for self, unable to carry on normal activity or to do active work; 100 , normal), who had received 1 or 2 previous regimens of anti-angiogenic therapy and no more than 3 of systemic therapy, and who experienced disease progression within 6 months of the start of the study, were enrolled. Patients with metastases to the brain or spinal cord, who received prior treatment with a mammalian target of rapamycin (mTOR) inhibitor, or who had a condition requiring glucocorticoid treatment, were excluded from the study.

Patients were randomized 1:1 with a block size of 4 to receive either nivolumab at a dose of $3 \mathrm{mg} / \mathrm{kg}$ body weight as a 60 -minute intravenous infusion every 2 weeks or the mTOR inhibitor everolimus administered orally at a daily dose of $10 \mathrm{mg}$. Randomization was stratified according to region (US or Canada, Western Europe, or rest of the world), Memorial Sloan Kettering Cancer Center prognostic risk group, and the number of previous anti-angiogenic treatment regimens. The MSKCC prognostic risk group is assessed on the basis of the presence of the prognostic factors anemia, hypercalcemia, and poor performance status

\section{What's new, what's important}

Renal cell carcinoma is the most common form of kidney cancer and because it is often diagnosed at a late stage when metastasis may have occurred, it is considered a difficult-to-treat disease. Until now, there were few treatment options, with everolimus, a mammalian target of rapamycin inhibitor, being the standard of care. The immunotherapeutic agent nivolumab was approved last fall for treatment of advanced clear-cell renal-cell carcinoma after anti-angiogenic therapy. It was shown to be more effective than everolimus and to extend overall survival $(25$ vs 19.6 months) across all specified groups. Findings for overall response rate and progression-free survival were also better with nivolumab. In addition, there was also demonstrated improvement in patient quality of life with nivolumab.

The most common treatment-related adverse effects with nivolumab were fatigue, nausea, stomatitis, and anemia, with $19 \%$ of patients experiencing grade $3 / 4$ TAEs (most common, fatigue; everolimus, $37 \%$, anemia) and $8 \%$ discontinuing because of TAEs (everolimus, 13\%). Prescribing information carries warnings for a number of immun-mediated sie-effects such as pneumonitis, colitis, hepatitis, endocrinopathy, and rash. The recommended dose of the medication for patients with advanced RCC is $3 \mathrm{mg} / \mathrm{kg}$ administered as a 60-minute IV infusion every 2 weeks until disease progression or unacceptable toxicity. It should be noted that nivolumab has not been studied in patients with moderate or severe hepatic impairment.

- Jame Abraham, MD, FACP (abrahaj5@ccf.org)

(0 factors, favorable risk; 1 , intermediate risk; 2 or 3 , poor risk). Demographics and clinical characteristics between the 2 treatment arms were well balanced.

Patients underwent computed-tomography or magnetic-resonance imaging at baseline, every 8 weeks for the first year, and then every 12 weeks until disease progression or discontinuation of treatment. They were followed for survival every 3 months after discontinuation. The primary endpoint was OS, and patients in the nivolumab arm had significantly improved OS compared with those in the everolimus arm (median OS, 25 vs 19.6 months; hazard ratio, $0.73 ; P=.002$ ) across all prespecified subgroups.

Secondary endpoints included objective response rate (ORR), progression-free survival (PFS), association

Report prepared by Jane De Lartigue, PhD. JCSO 2016;14:138-140. @2016 Frontline Medical Communications. doi: 10.12788/ jcso.0248. 


\section{Mechanism of action: nivolumab in RCC}

\section{Unleashing the immune system to prolong survival}

In recent years, the treatment landscape for advanced renal-cell carcinoma (RCC) has evolved markedly with anti-angiogenic therapies that target vascular endothelial growth factor becoming the standard of care in the front-line setting. Several second-line setting options are available following progression on these agents, including the mammalian target of rapamycin inhibitor everolimus. Although everolimus and other approved drugs greatly delay progression in this setting, there has been limited success in improving overall survival (OS).

As in other cancer types, studies have shown that RCC tumors often express programmed cell death ligand 1 (PD-L1), a protein that binds to and activates the programmed cell death 1 (PD-1) receptor found on the surface of $T$ cells, and patients whose tumors express PD-L1 have poorer prognosis. This sparked interest in testing immunotherapies that target the PD-1 receptor in RCC.

PD-1 is an inhibitory receptor that plays a role in the 2-step model of T-cell activation. The first step occurs when a $T$ cell encounters antigen-presenting cells (APCs) bearing foreign antigens, which engage the T-cell receptor. This is coupled with a second "costimulatory" signal, which completes T-cell activation and switches the T cell on, involving interaction between the CD28 receptor on the T cell and a costimulatory molecule on the surface of the APC.

To prevent inappropriate activation of $\mathrm{T}$ cells or to switch them off when they are no longer needed, which helps to avoid damage to healthy tissue, the second step can instead involve a "coinhibitory" signal that inactivates the T cell instead. The binding of the PD-1 receptor to its ligands PD-L1 and PD-L2 creates one example of this inhibitory signal. By expressing the PD-L1 protein on their surface, RCC tumors are able to engage the PD-1 receptor to switch off $T$ cells that enter the tumor microenvironment, which helps them to fly under the radar of the immune system.

Targeting PD-1 with monoclonal antibodies such as nivolumab,

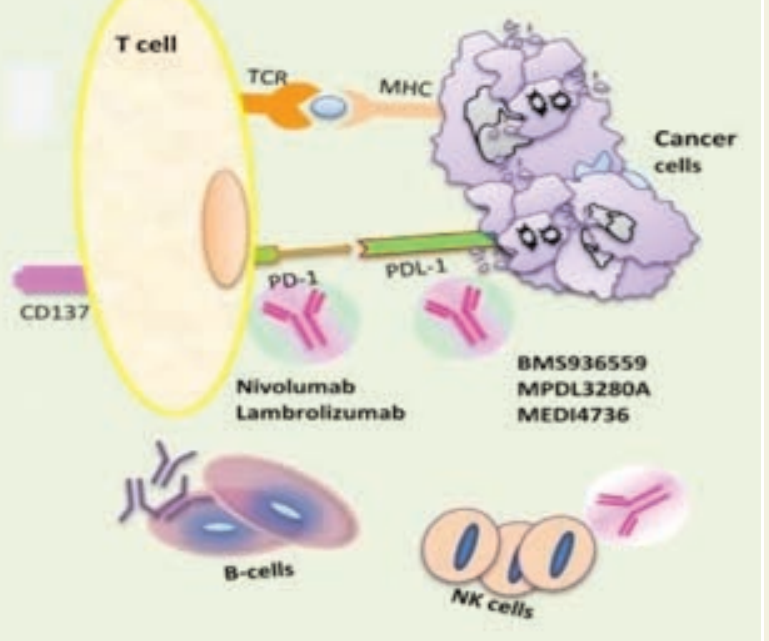

PD-L1 expressed on the surface of tumor cells engages the inhibitory PD-1 receptor on the surface of tumor-infiltrating $T$ cells and prevents T-cell activation. The use of immune checkpoint inhibitors, monoclonal antibodies that block PD-1 receptor activity (eg, nivolumab) or the ability of the PDL1 ligand to bind to the receptor (eg, MEDI4736) prevent downregulation of T-cell activity and help to reinstate the anti-tumor immune response.

Reproduced with permission: Gelao L, Criscitiello C, Esposito A, et al Immune Checkpoint Blockade in Cancer Treatment: A Double-Edged Sword Cross-Targeting the Host as an "Innocent Bystander". Toxins 2013;6(3): 914-933.

enables re-engagement of the anti-tumor immune response by boosting the activity of tumor-infiltrating $T$ cells. In the CheckMate-025 trial, nivolumab added to its track record, demonstrating significant anti-tumor efficacy in yet another tumor type, but most importantly providing an OS benefit for patients with advanced RCC in the second-line setting after failure of anti-angiogenic therapy. between OS and tumor expression of PD-L1, and incidence of adverse events (AEs). ORR, defined as the number of patients with complete or partial response (CR/PR) divided by the number of patients randomized, was also significantly improved in the nivolumab arm (25\% vs $5 \%$, respectively), with CRs in $1 \%$ and $\leq 1 \%$ of patients and PRs in $24 \%$ and $5 \%$. Median time to response was 3.5 months in the nivolumab arm, compared with 3.7 months in the everolimus arm and median duration of response was 12 months in both arms. Among patients who responded, these were ongoing at data cut-off of June 2015 in $48 \%$ and $45 \%$ of patients, respectively, with $31 \%$ and $27 \%$ of patients experiencing responses of 12 months or longer.

PFS was defined as the time from randomization to first documented RECIST-defined tumor progression or death from any cause. Median PFS was 4.6 months in the nivolumab arm and 4.4 months in the everolimus arm. The late separation of the PFS curves suggested a potential delayed PFS benefit for nivolumab. This was subsequently quantified in an ad hoc sensitivity analysis in patients who had not had disease progression or died at 6 months; median PFS was 15.6 months, compared with 11.7 months, respectively.

There was also a significant and consistent improvement in quality of life (QoL) with nivolumab, as evaluated by the Functional Assessment of Cancer Therapy Kidney Symptom Index - Disease-Related Symptoms (FKSI-DRS), which consists of 9 symptom-specific questions addressing lack of energy, pain, weight loss, bone pain, fatigue, dyspnea, cough, fevers, and hematuria. The median FKSI-DRS score in the 
nivolumab group improved over time and differed significantly from that observed in the everolimus group.

Among patients who had quantifiable tumor PD-L1 expression in pretreatment samples (92\%), 24\% of patients had expression levels of $\geq 1 \%$, and $76 \%$ had levels $<1 \%$. The OS benefit observed in the nivolumab group was independent of PD-L1 expression level of the tumor.

Dose delays were required in $51 \%$ of patients in the nivolumab arm and $66 \%$ of those treated with everolimus. Treatment-related AEs (TAEs) of any grade occurred in $79 \%$ and $88 \%$ of patients, respectively. The most common TAEs in the nivolumab arm were fatigue, nausea, and pruritus; and in the everolimus arm, fatigue, stomatitis, and anemia. The rate of grade $3 / 4$ TAEs was $19 \%$ in the nivolumab arm, most commonly fatigue, and $37 \%$ in the everolimus arm, most commonly due to anemia. TAEs led to discontinuation in $8 \%$ of nivolumab-treated patients and in $13 \%$ of everolimus-treated patients.

Nivolumab is marketed as Opdivo by Bristol-Myers Squibb. The prescribing information recommends a dose for patients with advanced RCC of $3 \mathrm{mg} / \mathrm{kg}$ administered as a 60 -minute intravenous infusion every 2 weeks until disease progression or unacceptable toxicity. No dosage adjustments are required for mild hepatic or renal impair-

\footnotetext{
References

1. Motzer RJ, Escudier B, McDermott DF, et al. Nivolumab versus everolimus in advanced renal-cell carcinoma. N Engl J Med. 2015;373:1803-1813.

2. Opdivo (nivolumab) injection, for intravenous use. Prescribing information. Bristol-Myers Squibb Company, January 2016. http://pack-
}

ment, but nivolumab has not been studied in patients with moderate or severe hepatic impairment.

The prescribing information provides warnings and precautions regarding the potential for a number of immunemediated side effects, including pneumonitis, colitis, hepatitis, endocrinopathy, and rash. A nivolumab infusion should be interrupted or slowed in patients with mild/ moderate infusion reactions and discontinued in the event of severe or life-threatening reactions. Nivolumab should be withheld for grade 2 pneumonitis, grade $2 / 3$ diarrhea or colitis, aspartate or alanine transaminase (AST/ALT) levels $>3-5$ times the upper limit of normal (ULN); total bilirubin $>1.5-3$ times ULN, serum creatinine $>1.5-6$ ULN, grade $2 / 3$ hypophytis, grade 2 renal insufficiency, grade 3 hyperglycemia, grade 3 rash, encephalitis, and first occurrence of other grade $3 \mathrm{AEs}$.

Nivolumab should be discontinued in the event of grade 4 diarrhea/colitis, grade 3/4 pneumonitis, grade 4 hypophytis, grade $3 / 4$ adrenal insufficiency, grade 4 rash, AST/ ALT $>5$ times ULN, total bilirubin $>3$ times ULN, serum creatinine $>6$ times ULN, encephalitis, in patients requiring prednisone, following any life-threatening or grade 4 $\mathrm{AE}$, recurrence of grade $3 \mathrm{AEs}$, or persistent grade $2 / 3 \mathrm{AEs}$ lasting $\geq 12$ weeks.

ageinserts.bms.com/pi/pi_opdivo.pdf. Accessed January 28, 2016.

3. FDA approves Opdivo to treat advanced form of kidney cancer. FDA news release. November 23, 2015. http://www.fda.gov/NewsEvents/ Newsroom/PressAnnouncements/ucm473971.htm. Accessed January $28,2016$. 